\title{
GENERALIZED HADAMARD MATRICES
}

\section{A. T. BUTSON}

1. Introduction. A square matrix $H$ of order $h$ all of whose elements are $p$ th roots of unity is called a Hadamard matrix $(H(p, h)$ matrix $)$ if $H H^{c T}=h I$. It is known [4] that $H(2, h)$ matrices can exist only for values $h=2$ and $h=4 t$, where $t$ is a positive integer. Although it has been conjectured that $H(2,4 t)$ matrices exist for all positive integers $t$, their existence has been established $[1 ; 3 ; 4 ; 5 ; 6 ; 7]$ for only the following values of $h$, where $q$ denotes an odd prime:

(1.1) $h=2^{k}$;

(1.2) $h=q^{k}+1 \equiv 0(\bmod 4)$;

(1.3) $h=h_{1}\left(q^{k}+1\right)$ where $h_{1} \geqq 2$ is the order of an $H(2, h)$ matrix;

(1.4) $h=h^{*}\left(h^{*}-1\right)$ where $h^{*}$ is a product of numbers of forms (1.1) and (1.2);

(1.5) $h=172$;

(1.6) $h=h^{*}\left(h^{*}+3\right)$ where $h^{*}$ and $h^{*}+4$ both are products of numbers of forms (1.1) and (1.2);

(1.7) $h=h_{1} h_{2}\left(q^{k}+1\right) q^{k}$ where $h_{1} \geqq 2, h_{2} \geqq 2$ are orders of $H(2, h)$ matrices;

(1.8) $h=h_{1} h_{2} s(s+3)$ where $h_{1} \geqq 2, h_{2} \geqq 2$ are orders of $H(2, h)$ matrices and where $s$ and $s+4$ both are of the form $q^{k}+1$;

(1.9) $h=(r+1)^{2}$ where both $r$ and $r+2$ are prime or prime powers;

(1.10) $h$ is a product of numbers of the forms (1.1)-(1.9). This list is taken from [2].

This paper is concerned with $H(p, h)$ matrices when $p>2$. The main result is the construction of $H\left(p, 2^{m} p^{k}\right)$ matrices where $p$ is a prime and $m \leqq k$ are non-negative integers.

2. Elementary properties. Some easily established results concerning $H(p, h)$ matrices which will be used in the sequel are the following:

(2.1) The requirement that $H H^{c T}=h I$ is equivalent to the requirement that $H^{C T} H=h I$; i.e., the orthogonality of any two rows of $H$ is equivalent to the orthogonality of any two columns of $H$.

(2.2) A permutation of the rows (columns) and multiplication of the elements of a row (column) by a fixed pth root of unity are elementary operations which leave invariant the Hadamard property.

(2.3) An $H(p, h)$ matrix can always be reduced to the standard form in which the initial row and column contain only the root 1.

Presented to the Society January 24, 1961; received by the editors June 9, 1961.

${ }^{1}$ It was noted by the referee that this result is known, and may be found in R. E. Bellman's Introduction to matrix analysis, McGraw-Hill, 1960, p. 27, problem 13. 
(2.4) If $H=\left(h_{i j}\right)$ is an $H(p, h)$ matrix in standard form, then

$$
\begin{aligned}
\sum_{j=1}^{h} h_{i j} & =\sum_{j=1}^{h} h_{i j}^{C}=0, & i & =2,3, \cdots, h ; \\
\sum_{i=1}^{h} h_{i j} & =\sum_{i=1}^{h} h_{i j}^{C}=0, & j & =2,3, \cdots, h .
\end{aligned}
$$

(2.5) If $H_{1}$ is an $H\left(p_{1}, h_{1}\right)$ matrix, $H_{2}$ is an $H\left(p_{2}, h_{2}\right)$ matrix, $h=h_{1} h_{2}$, and $p=1$. c.m. $\left(p_{1}, p_{2}\right)$, then $H_{1} \otimes H_{2}$ is an $H(p, h)$ matrix.

(2.6) If $H_{1}$ is an $H\left(p_{1}, h\right)$ matrix, $\gamma$ is a primitive $p_{2}$ th root of unity, and $p=1 . c . m .\left(p_{1}, p_{2}\right)$, then $\gamma H_{1}$ is an $H(p, h)$ matrix.

3. Construction of $H(p, h)$ matrices. Throughout the remainder of this paper $H$ will denote an $H(p, h)$ matrix in standard form and $\gamma$ a fixed primitive $p$ th root of unity.

When $p$ is a prime, the requirement (2.4) that $\sum_{j=1}^{h} h_{2 j}=0$ can be written in the form $\sum_{j=0}^{p-1} k_{j} \gamma^{j}=0$, where the $k_{j}$ are non-negative integers satisfying $\sum_{j=0}^{p-1} k_{j}=h$. Using $1=-\sum_{j=1}^{p-1} \gamma^{j}$, the condition becomes $\sum_{j=1}^{p-1}\left(k_{j}-k_{0}\right) \gamma^{j}=0$, where $\sum_{j=0}^{p-1} k_{j}=h$. Since $\gamma, \gamma^{2}, \cdots$, $\gamma^{p-1}$ are independent over the rational field, it is necessary that $k_{j}=k_{0}$ for $j=1,2, \cdots, p-1$. Hence $p k_{0}=h$ and the following result has been established.

THEOREM 3.1. When $p$ is a prime, an $H(p, h)$ matrix can exist only for values $h=p t$, where $t$ is a positive integer.

The necessary condition that $h=2$ or $h=4 t$ for $H(2, h)$ matrices has two obvious possible analogues for $H(p, h)$ matrices when $p$ is a prime; namely, $h=p$ or $h=p^{2} t$ and $h=p$ or $h=2 p t$. Results to follow in this section show that neither of these is necessary. The condition in the above theorem is the most stringent that has been obtained; and when $p$ is not a prime, even this is not necessary as the following immediate consequence of (2.6) shows.

TheOREM 3.2. It is possible to construct $H(2 p, h)$ matrices for $p$ arbitrary and $h$ any value described in (1.1)-(1.10).

By using (2.6) an $H(p, h)$ matrix can be constructed from an $H\left(p_{1}, h\right)$ matrix, where $p_{1}$ is a divisor of $p$. Such an $H(p, h)$ matrix can obviously be reduced by the elementary operations (2.2) to an $H\left(p_{1}, h\right)$ matrix; and, consequently, is considered as trivial.

Now let $V$ be the matrix defined by $v_{i j}=\gamma^{i j}, i, j=0,1, \cdots, p-1$. Then $\sum_{j=0}^{p-1} v_{i j} v_{k j}^{C}=\sum_{j=0}^{p-1} \gamma^{(i-k) j}$. When $i=k$, then $\sum_{j=0}^{p-1} \gamma^{(i-k) j}=p$. Suppose $i \neq k$. If $(i-k, p)=1$, then $\gamma^{i-k}$ is a primitive $p$ th root of 
unity and $\sum_{j=0}^{p-1} \gamma^{(i-k) j}=0$. If $(i-k, p)=d$ where $d>1$, let $p=p_{1} d$ and $i-k=i_{1} d$. Then $(i-k) p_{1}=i_{1} d p_{1}=i_{1} p \equiv 0(\bmod p)$, so that $\gamma^{i-k}$ is a $p_{1}$ th root of unity. In this case $\sum_{j=0}^{p-1} \gamma^{(i-k) j}=d \sum_{j=0}^{p_{1}-1} \gamma^{(\imath-k) j}=0$. This establishes the following theorem.

THEOREM 3.3. The Vandermonde matrix $V$ defined by $v_{i j}=\gamma^{i j}$, $i, j=0,1, \cdots, p-1$, is a symmetric $H(p, p)$ matrix. ${ }^{1}$

If $p=p_{1} p_{2} \cdots p_{r}$, where the $p_{j}$ are distinct prime powers, $\gamma_{j}$ is a primitive $p_{j}$ th root of unity, and $V_{j}$ the corresponding Vandermonde matrix, then permutation matrices $P$ and $Q$ exist such that $V=P\left(V_{1} \otimes V_{2} \otimes \cdots \otimes V_{r}\right) Q$. However, $V_{j}$ can not be so decomposed, so it would not have been sufficient to have proven the above theorem for $p$ a prime.

Suppose $p$ is odd, say $p=2 q+1$, and let $n$ be the smallest quadratic nonresidue of $p$. Denote by $U$ that permutation matrix such that $W=V U$ has elements $w_{i j}=\gamma^{n i j}, i, j=0,1, \cdots, p-1$. Define the matrix $Q$ by $q_{i j}=0$ for $i \neq j$ and $q_{i i}=\gamma^{q i^{2}}$ for $i=0,1, \cdots, p-1$. Then $C=Q V Q$ and $B=Q^{n} W Q^{n}$ are, by (2.2), $H(p, p)$ matrices. Using $-2 q \equiv 1(\bmod p)$, it is easy to see that $c_{i j}=\gamma^{q(i-j)^{2}}$ and $b_{i j}=\gamma^{n q(i-j)^{2}}$. Obviously now, $c_{i j}=c_{i+k, j+k}$ and $b_{i j}=b_{i+k, j+k}$ for $k=0,1, \cdots, p-1$, so that $C$ and $B$ are cyclic matrices. Furthermore, $C$ and $B$ are symmetric matrices, and each contains at most $q+1$ distinct $p$ th roots of unity.

Defining the product of two rows $v_{i}$ and $v_{j}$ of $V$ to be that vector obtained by multiplying $(\bmod p)$ the corresponding components of the two rows, it is noted that $v_{i} v_{j}=v_{i+j}$, so that the rows of $V$ form a cyclic group with generator $v_{1}$. Similarly, the columns of $V$, the rows of $W$, and the columns of $W$ all form cyclic groups with generators $v_{1}^{T}, w_{1}=v_{n}$, and $w_{1}^{T}=v_{n}^{T}$, respectively. From this observation it easily follows that $D^{k} V=V T^{k}$ and $D^{n k} W=W T^{k}$, where $D$ is the matrix defined by $d_{i j}=0$ for $i \neq j$, and $d_{i i}=\gamma^{i}$ for $i=0,1, \cdots, p-1$, and $T$ is the permutation matrix defined by $t_{i+1, i}=1$ for $i=0,1, \cdots, p-1$ and $t_{i j}=0$ otherwise.

Let $Y=(11 \cdots 1)$ and $Z=(00 \cdots 0)$, both of length $p$. Then the $k$ th column of $B$ can be written in the form $T^{k} Q^{n} Y^{T}$, and the $k$ th column of $C P$ in the form $T^{k n} Q Y^{T}$. It will now be easy to prove the following construction theorem.

Theorem 3.4. When $p$ is a prime, an $H(p, 2 p)$ matrix can be constructed.

The procedure will be to show that the matrix 


$$
K=\left(\frac{Q V}{(C P)^{C T}} \mid \frac{Q^{n} W}{B^{C T}}\right)
$$

is an $H(p, 2 p)$ matrix. First it is noted that $C Y^{T}=\left(Y Q Y^{T}\right) Y^{T}$ and $B Y^{T}=\left(Y Q^{n} Y^{T}\right) Y^{T}$. When $p=2 q+1$ is prime, there are $q$ quadratic residues and $q$ quadratic nonresidues of $p$. Consequently,

$$
Y Q Y^{T}+Y Q^{n} Y^{T}=\sum_{i=0}^{p-1} \gamma^{q i^{2}}+\sum_{i=0}^{p-1} \gamma^{n q i^{2}}=2 \sum_{j=0}^{p-1} \gamma^{q j}=0 .
$$

Thus $C Y^{T}+B Y^{T}=Z^{T}$. Now $K K^{C T}$ in block form is

$$
\left(\frac{(Q V)(Q V)^{C T}+\left(Q^{n} W\right)\left(Q^{n} W\right)^{C T}}{(C P)^{C T}(Q V)^{C T}+B^{C T}\left(Q^{n} W\right)^{C T}} \mid \frac{(Q V)(C P)+\left(Q^{n} W\right) B}{(C P)^{C T}(C P)+B^{C T} B}\right) .
$$

By (2.2), $Q V, Q^{n} W, C P$, and $B$ are all $H(p, p)$ matrices. Thus $(Q V)(Q V)^{C T}+\left(Q^{n} W\right)\left(Q^{n} W\right)^{C T}=(C P)^{C T}(C P)+B^{C T} B=2 p I_{p}$. Now consider $(Q V)(C P)+\left(Q^{n} W\right) B$. Using the fact that the $k$ th columns of $C P$ and $B$ can be written as $T^{k n} Q Y^{T}$ and $T^{k} Q^{n} Y^{T}$, respectively, the $k$ th column of $(Q V)(C P)+\left(Q^{n} W\right) B$ is then given by

$$
\begin{aligned}
Q V T^{k n} Q Y^{T}+Q^{n} W T^{k} Q^{n} Y^{T} & =D^{k n} Q V Q Y^{T}+D^{k n} Q^{n} W Q^{n} Y^{T} \\
& =D^{k n}\left(C Y^{T}+B Y^{T}\right)=D^{k n} Z^{T}=Z^{T} .
\end{aligned}
$$

Hence, $(Q V)(C P)+\left(Q^{n} W\right) B$ and its conjugate transpose $(C P)^{C T}(Q V)^{C T}+B^{C T}\left(Q^{n} W\right)^{C T}$ are both 0 . Thus $K K^{C T}=2 p I_{2 p}$ and the theorem is proven. An immediate consequence of this theorem and (2.5) is now stated.

Theorem 3.5. When $p$ is a prime, $H\left(p, 2^{m} p^{k}\right)$ matrices can be constructed for any non-negative integers $m \leqq k$.

All the preceding results on the construction of $H(p, h)$ matrices are summarized in the following theorem.

TheOREM 3.6. Let $p=2^{k_{0}} p_{1}^{k_{1}} p_{2}^{k_{2}} \cdots p_{r}^{k_{r}}$ be the factorization of $p$ into powers of distinct primes. If $k_{0}=0$, then $H\left(p, h_{1}\right)$ matrices can be constructed for $h_{1}=2^{j_{0}} p_{1}^{j_{1}} p_{2}^{j_{2}} \cdots p_{r}^{j_{r}}$, where $j_{i} \geqq 0, i=0,1, \cdots, r ; j_{i}>0$ for at least one $i>0 ;$ and $j_{0} \leqq \sum_{i=1}^{r} j_{i}$. If $k_{0} \neq 0$, then $H\left(p, h_{4}\right)$ matrices can be constructed for $h_{4}=h_{2} h_{3}$, where $h_{2}$ is 1 or the order of any $H(2, h)$ matrix, and $h_{3}$ is 1 or any value of $h_{1}$.

4. Remarks. Let the matrix obtained from $H$ by deleting the initial row and column of 1's be called the core of $H$. Let $\pi$ be a primitive root of the prime $p$. Then there exists a permutation matrix $P$ such 
that the core of $P V P$ is the cyclic matrix whose rows are all the cyclic permutations of $\left(\gamma^{\pi} \gamma^{\pi^{2}} \cdots \gamma^{\pi^{p-1}}\right)$. The rows of $P V P$ obviously form a group. In a subsequent paper the connection between an $H\left(p, p^{n}\right)$ matrix whose rows form a group and whose core is cyclic, a maximal length linear recurring sequence with elements in $G F(p)$, and a "relative" difference set will be shown. One consequence of this connection is the following theorem.

THEOREM 4.1. For any prime $p$ and any positive integer $n$, an $H\left(p, p^{n}\right)$ matrix whose rows form a group and whose core is cyclic can be constructed.

\section{REFERENCES}

1. R. C. Bose, On the construction of balanced incomplete block designs, Annals of Eugenics 9 (1939), 353-399.

2. R. C. Bose and S. S. Shrikhande, A note on a result in the theory of code construction, Information and Control 2 (1959), 183-194.

3. A. Brauer, On a new class of Hadamard determinants, Math. Z. 58 (1953), 219225.

4. R. E. A. C. Paley, On orthogonal matrices, J. Math. Phys. 12 (1933), 311-320.

5. R. G. Stanton and D. A. Sprott, A family of difference sets, Canad. J. Math. 10 (1958), 73-77.

6. J. Williamson, Hadamard detcrminant theorem and sum of four squares, Duke Math. J. 11 (1944), 65-81.

7. - Note on Hadamard's determinantal problem, Bull. Amer. Math. Soc. 53 (1947), 608-613.

Boeing Airplane Company 